\title{
A DESCRIPTIVE STUDY OF PERIOPERATIVE COMPLICATIONS IN PATIENTS UNDERGOING PERCUTANEOUS NEPHROLITHOTRIPSY UNDER GENERAL ANAESTHESIA
}

\author{
Santhi K. S1, Jithin Mathew Abraham², Mary Albert ${ }^{3}$
}

${ }_{1}^{1}$ Additional Professor, Department of Anaesthesiology, Government Medical College, Kottayam.

${ }^{2}$ Assistant Professor, Department of Anaesthesiology, Believers Church Medical College, Thiruvella.

3 Professor, Department of Anaesthesiology, Pushpagiri Medical College, Thiruvella.

\begin{tabular}{l}
\hline ABSTRACT \\
BACKGROUND \\
Percutaneous Nephrolithotripsy is a commonly employed surgical technique in handling renal calculi. Because of the nature of the \\
surgery and especially the use of irrigating fluid, patients may develop various complications like haemodynamic instability, fluid \\
and electrolyte imbalance etc. Better understanding of these complications help improve patient care and thus patient outcome. \\
The objective of this study is to study the occurrence of perioperative complications associated with PCNL under balanced \\
general anaesthesia in prone position using distilled water as irrigation solution and the possible risk factors involved.
\end{tabular}

\section{MATERIALS AND METHODS}

75 patients scheduled for PCNL were randomly chosen for the study and each patient was assessed for co-morbid illness, electrolyte and renal function abnormalities. Standard balanced general anaesthesia was provided and the patient was positioned prone for the procedure. Following recovery from anaesthesia, HR, BP, haematocrit along with serum sodium and potassium levels were estimated.

Statistical Analysis- To analyse the proportions, Chi-square test was used for qualitative variables and paired t-test for quantitative variables.

Setting and Design- Descriptive study.

\section{RESULTS}

Out of 75 patients $76 \%$ developed hypertension, $16 \%$ had hypertension at induction, $40 \%$ during intraoperative period and $20 \%$ in the postoperative period. Tachycardia was noted in $24 \%, 35 \%$ and $16 \%$ of patients during induction, intraoperative period and postoperative periods respectively. Preoperative mean haemoglobin was 13.8 gm\% and postoperative mean $\mathrm{Hb}$ was 12.45 and the change was significant. The mean preoperative sodium level was 138.5 and postoperative value was 136.7, the change being statistically significant. Potassium did not show a significant change with the mean values before and after the procedure, 4.14 and 4.13 respectively. $60 \%$ of the patients did not have any problems. $15 \%$ had delayed recovery, while $5 \%$ had hypertension with tachycardia; $5 \%$ of the patients had airway obstruction and 1 patient had hydropneumothorax.

\section{CONCLUSION}

Percutaneous nephrolithotripsy is associated with significant increase in blood pressure and heart rate along with a reduction in the sodium levels.

\section{KEYWORDS}

Perioperative Complications, Percutaneous Nephrolithotomy, Hypertension, Haemoglobin, Hyponatraemia, Delayed Recovery. HOW TO CITE THIS ARTICLE: Santhi KS, Abraham JM, Albert M. A descriptive study of perioperative complications in patients undergoing percutaneous nephrolithotripsy under general anaesthesia. J. Evolution Med. Dent. Sci. 2017;6(94):6845-6848, DOI: $10.14260 /$ jemds $/ 2017 / 1483$

\section{BACKGROUND}

Percutaneous Nephrolithotomy (PCNL) is a minimal invasive surgery for renal calculi that was first reported by Fernstrom and Johansson in 1976.(1) Most urologists believe that this operation is better than open surgery as it decreases the length of stay, less morbidity, less pain and more preserved kidney function.(2)

Several changes and modifications have taken place in the last few years in an attempt to further refine the procedure

'Financial or Other Competing Interest': None.

Submission 21-04-2017, Peer Review 26-11-2017,

Acceptance 04-12-2017, Published 18-12-2017.

Corresponding Author:

Dr. Jithin Mathew Abraham,

Assistant Professor

Department of Anaesthesiology,

Believers Church Medical College, Thiruvella.

E-mail: jithinmab@gmail.com

DOI: $10.14260 /$ jemds $/ 2017 / 1483$ and to lower the morbidity, analgesic requirements and duration of hospitalisation. These include the use of regional blocks, single step dilatation, "Mini-Perc" technique, tubeless PCNL and sandwich therapy.(3),(4),(5) PCNL is mostly performed under general anaesthesia.(5) The objective of the present study is to evaluate the occurrence of perioperative complications with regard to haemodynamic changes, haematocrit and electrolyte changes and postoperative recovery for a patient undergoing PCNL under general anaesthesia in prone position with distilled water as irrigation fluid and also to study the risk factors associated.

\section{Background and Rationale}

PCNL is a common urological procedure and is the treatment of choice for staghorn and multiple pelvic calculi. Patients are prone for complication in the perioperative period due to multiple patient related, procedure related and anaesthesia related factors. According to the literature, the total complication rate of PCNL was found to be $83 \% .^{(6-8)}$ Many of 
the untoward events can be avoided by anticipation of the problem, meticulous preparation in each step and by adopting early corrective measures.

Study Design- Descriptive study.

Period of Study- 6 months.

\section{Location}

Dept. of Anaesthesiology, Govt. Medical College, Kottayam.

\section{Sample Size (Calculated)- 75.}

The variables for calculation is derived from a previous study by Vorrakit et al, in which the cardiovascular complications contributed to $57.1 \%$.

Formula Used: $4 \times P \times Q \div L \quad 2$

$4=$ square of ' $z$ ' value for alpha error at $5 \%$

$\mathrm{P}=$ proportion in the reference study (57.1)

$\mathrm{Q}=100-\mathrm{P}=42$

$\mathrm{L}=$ relative precision $(20 \%$ of 57.1$)$

\section{MATERIALS AND METHODS}

After obtaining the Ethical Committee clearance, 75 patients scheduled for PCNL were randomly chosen for the study and each patient was assessed for comorbid illness, electrolyte and renal function abnormalities. Standard balanced general anaesthesia was provided using Midazolam (1 $\mathrm{mg}$ ) and glycopyrrolate $(0.2 \mathrm{mg})$ as premedication and Propofol $(2$ $\mathrm{mg} / \mathrm{kg}$ ) for induction along with Fentanyl (2 ug/kg) and Vecuronium $(0.1 \mathrm{mg} / \mathrm{kg})$ and patient was positioned prone for the procedure. Following recovery from anaesthesia, HR, $\mathrm{BP}$ and haematocrit along with serum sodium and potassium levels were estimated.

\section{Statistical Analysis}

To analyse (using SPSS-16) the proportions, Chi-square test was used for qualitative variables and paired t-test for quantitative variables.

\section{RESULTS}

Out of the 75 patients who underwent PCNL, 77\% were males and $23 \%$ were females and the mean age was 46.24 years with a standard deviation of 11.8 years. Among the above sample, $75 \%$ had preoperative renal dysfunction and $28 \%$ had hypertension requiring medical therapy. The mean duration of surgery was 47.8 minutes with a standard deviation of 21.6 and the average volume of the irrigation fluid used was 16.2 litres with a standard deviation of 7.7 litres.

\section{Haemodynamic Changes}

Out of the 75 patients, $16 \%$ had hypertension at induction, $40 \%$ had high blood pressure during the intraoperative period, while $20 \%$ had postoperative hypertension. Tachycardia was noted in $24 \%, 35 \%$ and $16 \%$ of patients during induction, intraoperative period and postoperative periods respectively. Out of the 75, 33 non-hypertensive patients developed rise in BP and heart rate more than $20 \%$ of the baseline.

\begin{tabular}{|c|c|c|c|}
\hline & Induction & Intra-op & Post-op \\
\hline Hypertension & $16 \%$ & $14 \%$ & $20 \%$ \\
\hline Tachycardia & $24 \%$ & $35 \%$ & $16 \%$ \\
\hline
\end{tabular}

\section{Change in Haemoglobin Levels}

The preoperative mean haemoglobin level was 13.8 and postoperative mean was 12.45 with a ' $t$ ' value of 8.64 and a ' $p$ ' value of $<0.001$, both of which are statistically significant. The changes are correlated with duration of procedure, amount of irrigation fluid and procedure related injuries.

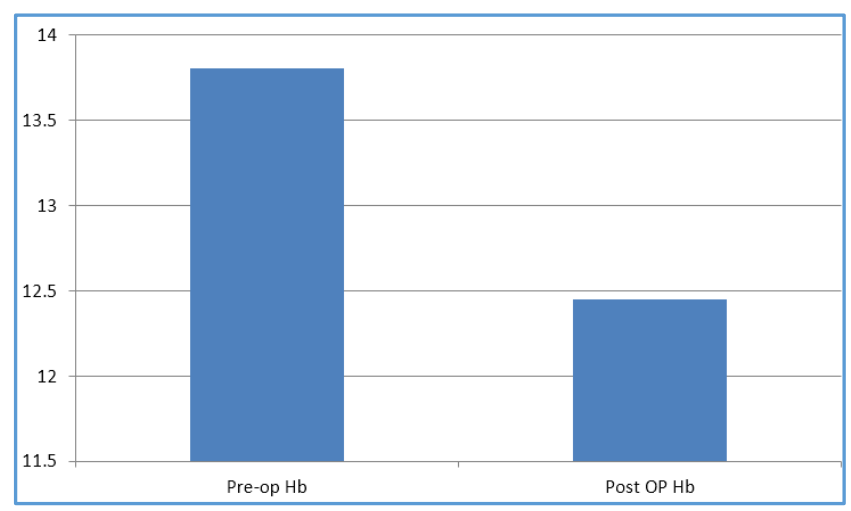

Figure 1. Showing Pre-Operative and Post-Operative Hb

\section{Changes in Electrolytes}

The mean level of serum Na levels in the pre-op period is 138.5 and that in the post-op period is 136.7 , where the ' $t$ ' value is 3.37 and ' $p$ ' value is 0.001 . The change in $\mathrm{Na}$ levels is statistically significant.

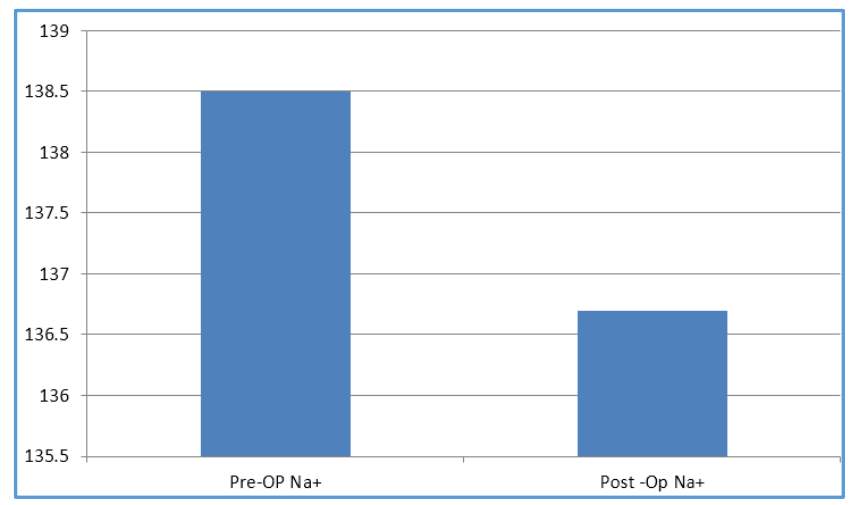

Figure 2. Showing Pre-Operative and Post-Operative Sodium Levels

Potassium did not show a significant change with the mean values before and after the procedure, 4.14 and 4.13 respectively.

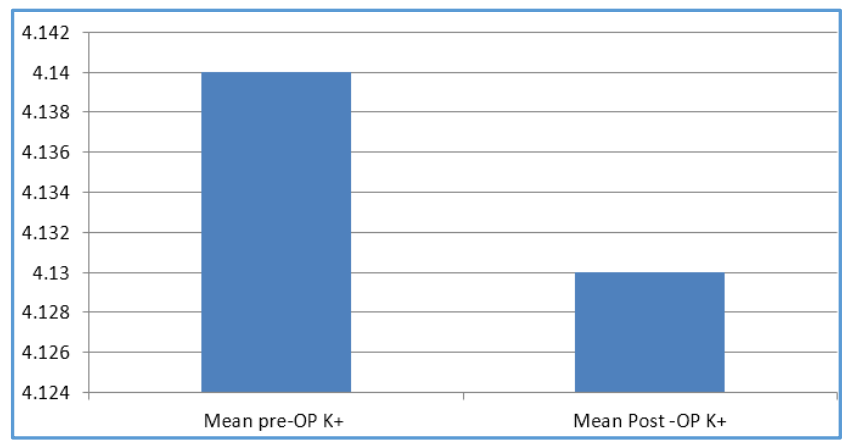

Figure 3. Showing Mean Potassium Values before and after Surgery 


\section{Post-Operative Complication}

$60 \%$ of the patients did not have any problems. $15 \%$ had delayed recovery, while $5 \%$ had hypertension with tachycardia. $5 \%$ of the patients had airway obstruction. $3 \%$ had pain and 1 patient had hydropneumothorax.

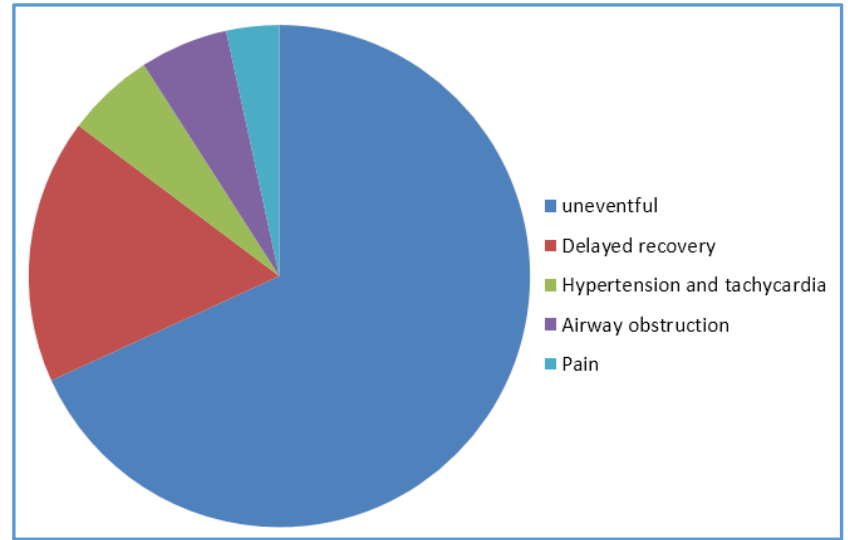

Figure 4. Shows Incidence of various Perioperative Complications

\section{Correlation between Perioperative Complications and Hypertension}

Among the hypertensive patients only $38.1 \%$ had complications, while among the non-hypertensives $70.4 \%$ had one or the other complication. The difference is statistically significant as evidenced by a 'p' value of 0.01 and Chi-square test of 6.64 .

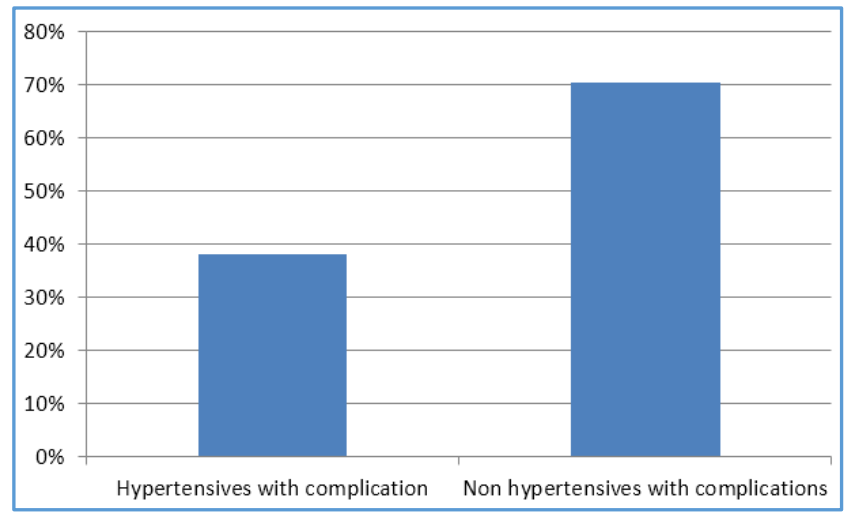

\section{DISCUSSION}

PCNL is the treatment of choice for large kidney stones, staghorn calculi, multiple stones resistant to ESWL.(9) PCNL is better than open surgery in terms of morbidity, hospital stay and procedure related complications.(10)

PCNL is done under general anaesthesia or regional anaesthesia with patient in prone position. The perioperative complications associated with PCNL is due to anaesthesia related, procedure related, irrigation fluid related and patient related factors.

The study by Mehrabi et al(9) failed to demonstrate any advantage of spinal anaesthesia over general anaesthesia, while Tangy Paitoon et al(11) demonstrated the beneficial effects of epidural anaesthesia in PCNL. Kuzgunbay et al(12) studied the perioperative complications and risk factors associated with PCNL using normal saline as irrigation fluid.

It is very difficult to totally eliminate the complication associated with PCNL. The nature of the procedure, pre- existing renal dysfunction and co-existing systemic illnesses make PCNL prone for complications.

The various perioperative complications observed may be prevented by adopting meticulous anaesthesia techniques and considering factors like pre-existing renal dysfunction, hypertension, obesity and difficult airway. Complications were more among non-hypertensives compared to hypertensive patients. This observed change in haemodynamics may be due to suppression of stress response in the perioperative period in hypertensives due to intake of drugs like beta blockers and calcium channel blockers. Adequate doses of sedative premedication, measures to prevent stress response and anticipatory preparations to tackle difficult airway will prevent excessive haemodynamic variations at induction. During the procedure in prone position, the stimulation of kidneys and pelvis need to be meticulously suppressed by maintaining a deep plane of anaesthesia using volatile agents or epidural analgesia. In our study, both of these were not used.

The problem of hyponatraemia in our study is related to the use of distilled water and is also directly related to the duration of the procedure. Use of normal saline in place of distilled water is recommended to prevent this. The problem of electroconductivity as in the case of TURP does not exist in PCNL and change over to normal saline is beneficial. In our study, change in potassium values was not significant. A rise in potassium will lead to exaggeration of cardiac toxicity in the setting of hyponatraemia.

In this study, changes in haemoglobin levels were noted which is due to blood loss during the procedure and haemodilution by the absorbed fluid. Limiting the duration of procedure and care to avoid inadvertent bleeding from the renal parenchyma helps in reducing postoperative anaemia.

Recovery related problems are mostly avoidable. Use of short-acting hypnotics and opioids and use of Epidural analgesia helps improve recovery.

\section{CONCLUSION}

Percutaneous nephrolithotripsy is associated with significant increase in blood pressure and heart rate along with a reduction in the sodium levels. Potassium levels had no significant variation.

\section{REFERENCES}

[1] Sebnem A, Zeren S, Aribogan A. Hormonal and hemodynamic changes during percutaneous nephrolithotomy. Internat Urol \& Nephrology 2001;32(3):311-4.

[2] Koroglu A, Togal T, Cicek M, et al. The effects of irrigation fluid volume and irrigation time on fluid electrolyte balance and hemodynamics in percutaneous nephrolitho-tripsy. Internat Urol Nephrology 2003;35(1):1-6.

[3] Goh M, Walf JS. Almost totally tubeless percutaneous nephrolithotomy: further evolution of the technique. Journal Endourology 1999;13(3):177-80.

[4] Said MM, Sonbul ZM, El-Kenaway M, et al. Spinal and interpleural bupivacaine for percutaneous nephrolithotomy. Middle East J Anesthesiology 1991;11(3):259-64. 
[5] Jackman SV, Hedican SP, Peter CA, et al. Percutaneous nephrolithotomy in infants and preschool age children: experience with a new technique. Urology 1998;52(4):697-701.

[6] Netto NR, Ikonomidis J, Ikari O, et al. Comparative study of percutaneous access for staghorn calculi. Urology 2005;65(4):659-63.

[7] Kim SC, Kuo RL, Lingeman JE. Percutaneous nephrolithotomy: an update. Curr Opin Urol 2003;13(3):235-41.

[8] Liatsikos EN, Kapoor R, Lee B, et al. "Angular percutaneous renal access". Multiple tracts through a single incision for staghorn calculous treatment in a single session. Eur Urol 2005;48(5):832-7.

[9] Mehrabi S, Shirazi KK. Results and complications of spinal anesthesia in percutaneous nephrolithotomy. Urol J 2010;7(1):22-5.
[10] Kumar A, Verma BS, Gogoi S, et al. A prospective randomized trial of open surgery versus endourological stone removal in patients of staghorn stones with chronic renal failure. Indian J Urol 2001;18(1):14-9.

[11] Tangpaitoon T, Nisoog C, Lojanapiwat B. Efficacy and safety of percutaneous nephrolithotomy (PCNL): a prospective and randomized study comparing regional epidural anesthesia with general anesthesia. Int Braz J Urol 2012;38(4):504-11.

[12] Kuzgunbay B, Turunc T, Akin S, et al. Percutaneous nephrolithotomy under general versus combined spinal-epidural anesthesia. Endourol 2009;23(11):1835-8. 